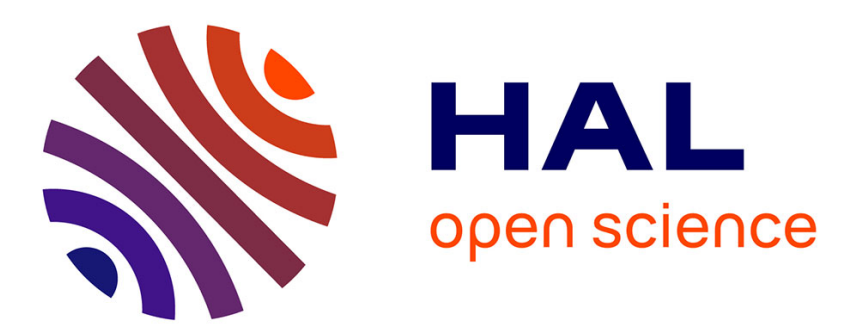

\title{
An analytical modeling with experimental validation of bone temperature rise in drilling process
}

\author{
Foli Amewoui, Gael Le Coz, Anne-Sophie Bonnet, Abdelhadi Moufki
}

\section{To cite this version:}

Foli Amewoui, Gael Le Coz, Anne-Sophie Bonnet, Abdelhadi Moufki. An analytical modeling with experimental validation of bone temperature rise in drilling process. Medical Engineering \& Physics, 2020, 84, pp.151-160. 10.1016/j.medengphy.2020.07.007 . hal-03040692

\section{HAL Id: hal-03040692 \\ https://hal.univ-lorraine.fr/hal-03040692}

Submitted on 4 Dec 2020

HAL is a multi-disciplinary open access archive for the deposit and dissemination of scientific research documents, whether they are published or not. The documents may come from teaching and research institutions in France or abroad, or from public or private research centers.
L'archive ouverte pluridisciplinaire HAL, est destinée au dépôt et à la diffusion de documents scientifiques de niveau recherche, publiés ou non, émanant des établissements d'enseignement et de recherche français ou étrangers, des laboratoires publics ou privés. 


\section{An analytical modelling with experimental validation of bone} temperature rise in drilling process

Foli Amewoui, Gaël Le Coz, Anne-Sophie Bonnet ${ }^{\star}$, Abdelhadi Moufki

4 Laboratoire d'Etude des Microstructures et de Mécanique des Matériaux, LEM3

$5 \quad$ Université de Lorraine, CNRS, Arts et Métiers Paris Tech, LEM3, F-57000 Metz, 6

France

7

8

9 foli-noel.amewoui-ekoue-adjoka@univ-lorraine.fr;

10 gael.lecoz@univ-lorraine.fr

11 abdelhadi.moufki@univ-lorraine.fr

12

$13{ }^{*}$ Corresponding author: Anne-Sophie Bonnet

14 e-mail: anne-sophie.bonnet@univ-lorraine.fr,

15 tel: +33 (0)3-72-74-86-47

16

17 


\section{ABSTRACT}

Predicting the bone thermal response in a surgical operation remains a major challenge. In the previous works, metal machining theory has frequently been used to predict bone temperature in drilling process. However, several experimental studies demonstrate that the chip formation process is very complex compared to metal cutting. In the present study, a simplified analytical model based on the moving heat source approach combined with the method of image sources is developed. The heat source due to the drill-bit tip was supposed to be proportional to the cutting energy. The friction at the tool-hole contact was also considered. An experimental study was performed on fresh femur pig bone for cutting speeds from 2 to $20 \mathrm{~m} / \mathrm{min}$. Temperature rise, drilling forces and bone volume fraction were measured. The experimental validation showed that the model reproduces satisfactorily the increase in temperature up to the maximum value while it overestimates the temperature during the cooling stage. A parametric study (thermal boundary conditions, lateral friction) was also performed. From the predicted results, it appears that the model can be improved by considering the effects of the bone volume fraction which can present a significant variation in the bone sample.

\section{Keywords}

Bone drilling, Bone necrosis, Bone temperature rise, Thermal model for bone drilling, Moving heat source, Axial drilling force and torque, Bone volume fraction.

\section{INTRODUCTION}

Bone cutting is a common step in bone fracture treatment and implantology. Notwithstanding that bone cutting method includes grooving, sawing, drilling, and milling, the most discussed process in the literature is bone drilling [1]. The reason of 
this increasing interest among researchers comes from the fact that drilling process generates significant heat that may lead to thermal injury of bone [2-4]. Hillery and Shuaib [5] report that, for exposure time greater than $30 \mathrm{~s}$, the necrosis threshold in human and bovine bones is $55^{\circ} \mathrm{C}$. According to Bertollo et al. [6], Pandey and Panda [1], bone necrosis can appear for very small exposure time when the bone temperature is $70^{\circ} \mathrm{C}$. In spite of the fact that there is no consensus concerning the critical values of exposure duration and temperature magnitude, the typical value of $47^{\circ} \mathrm{C}$ during at least $60 \mathrm{~s}$ is often used as a reference threshold for the thermal injury apparition [3,7].

Pioneering works related to bone drilling deal about the prevention of osteonecrosis. The key step of these investigations is the assessment of the cutting temperature by using experimental and/or theoretical techniques [1,3,8-10]. Due to the variability of bone samples properties and experimental conditions and to the large number of drilling parameters, these investigations often do not converge towards general conclusions about the temperature rise with respect to the drilling parameters. So, it appears necessary to develop realistic thermo-mechanical models of bone drilling operation to investigate the effect of each parameter, on temperature elevation.

Metal machining theory has frequently been used to calculate drilling heat generated during bone drilling $[8,11,12]$. The authors assumed that bone behaves like metal when it is machined. Therefore, the deformation in the chip was supposed to be characterized by three zones; the primary deformation zone where the required energy in material shearing is converted into heat; the secondary zone where heat is generated by shearing along the tool rake face and the third zone where friction between the tool clearance face and the new surface of bone produces heat. A similar assumption has been taken by Lee et al. [8] to suggest a thermal model for bone drilling. In their investigation, the heat generated in the third zone was assumed to be 
negligible for a sharp cutting tool, exactly as in the case of metal cutting. This theory has been extended further upon by Maani et al. [12] to develop a mathematical model for predicting the thermal response in the surgical drilling of bone. To determine the temperature rise during drilling, Kalidindi [13] also used the approach developed by Oxley [14].

Other authors investigated the cutting and chip formation mechanism of bone cutting and suggested an alternative view. Jacob et al. [15] analysed, through microscopic and SEM images, bone chips shape during orthogonal cutting. They showed that Merchant [16] analysis of orthogonal cutting, commonly used in material cutting, has limited applicability to bone material. Further investigations done by Wiggins and Malkin [17] on the mechanics of orthogonal machining of bone showed that the chip formation process occurred by a series of discrete fractures. Recently, Liao and Axinte [18] identified three different chip formation modes related to the uncut chip thickness. Feldman et al. [19] corroborated the observations of Liao and Axinte [18]. They found the bone cutting behaviour changed from a ductile to a fracture cutting mode as cutting depth increases. The threshold cutting depth inducing cracks with chips formation was shown to be $20 \mu \mathrm{m}[20]$.

Thus, within these experimental investigations, it appears that the chip formation process is very complex compared to metal cutting and that during bone cutting process, chips are formed more by fracture mode than shear mode, regardless of cutting direction, as shown in Fig. 1. Consequently, it seems more realistic to assume that bone behaves like a quasi-brittle material in drilling process. Therefore, whereas for metal cutting the heat sources in the chip are clearly associated to the plastic deformation in the shear zones, the temperature elevation inside bone can clearly not be associated to the same physical mechanisms. As a consequence, analytical models 
based on metal machining theory cannot be considered as an appropriate approach to predict the bone temperature rise in drilling. In addition, the source of the heat generated during bone drilling is not clearly defined for a wide range of drilling conditions and needs further investigation.

Recently, new thermal models for bone drilling, based on the moving heat source approach of Jaeger [21], have been introduced by Feldmann et al. $[19,22]$. The interactions between drill tip and bone are modeled by a moving heat source assimilated to a disk source with the size of the drill bit diameter. The authors $[19,22]$ expressed the thermal energy as a function of bone density distribution. With the Green's function of time-dependent moving point source, they determined the temperature distribution in function of time. It can however be noted that the model $[19,22]$ presents different limitations: (i) the bone has been assimilated to an infinite medium (i.e. the bone material fills all the space). As it will be shown in the present work, this assumption may induce an underestimation of bone temperature if the feed rate is not large enough. (ii) during the drill bit penetration into the bone, the distance between the heat source, which moves with the tool, and the measurement position of temperature has been taken constant for a fixed time $t$, (iii) the heat sources induced by the bone-tool interactions are restricted to the tool tip effect.

As indicated previously, the bone behaves as a quasi-brittle material during cutting process. Therefore, friction between the drilled hole and the tool can affect the bone temperature. In the present work, a simplified analytical model is developed using the following assumptions: (i) bone temperature rise results from two main sources corresponding to the interaction between the drill-bit tip and bone and the friction between the drill-bit side and the drilled hole, (ii) the effect of the point angle is neglected and the heat source associated to the tool tip is assimilated to a disk. The 
119 model was used to analyze the influence of spindle speed which affects the heat

120 generation and the heat transfer through the exposure time. Experimental tests of bone

121 drilling have also been conducted on porcine samples. After a validation study of the

122 model with temperature experimental data, a parametric study has been performed

123 and compared with the results of the experimental campaign.

$125 \quad 2 . \quad$ MATERIALS AND METHODS

126

\subsection{Experimental study}

127 Bone samples used in these experiments were extracted from the diaphyseal part of a porcine femoral bone aged of 6 months. The bone was obtained from a local slaughterhouse just after the slaughter of the animal and directly frozen $\left(-20^{\circ} \mathrm{C}\right)$. There was no animal sacrifice specifically for this study. Experiments were conducted on

131 bones devoted to food industry. Before sample preparation, bone was thawed to a

132 temperature between 3 and 6 degrees during 24 hours. Bone samples were finally 133 machined at room temperature to get dimensions of approximately $70 \mathrm{~mm}$-long and

$13410 \mathrm{~mm}$-wide. Fig. 2(a) shows the final state of the sample to drill.

136 Each bone sample was scanned by X-ray micro-computed tomography $(\mu \mathrm{CT})$ machine

137 EasyTom (RX Solutions, Chavanod, France) before drillings tests. The scanning parameters are the following ones: voltage $100 \mathrm{keV}$, current $50 \mu \mathrm{A}$, exposure time 2.6 s and voxel size $15 \times 15 \times 15 \mu \mathrm{m}^{3}$. Microtomography was conducted on several bone

140 samples but the most homogeneous sample in terms of microstructure (i.e. with a 141 cortical layer of quite constant thickness along the whole specimen) and presenting 
142 the highest thickness of cortical bone was kept for the drilling process and the

143 comparison with the analytical model.

144 After image acquisition of the bone sample, the drilling experiments were performed

145 on it. A feed per tooth of $0.02 \mathrm{~mm}$ was chosen and cutting velocities varying between

1462 to $20 \mathrm{~m} / \mathrm{min}$ were tested. The drilling tests were performed on a three axes CNC

147 milling machine Roeders RP600. A Nobel Biocare twist drill of $3.2 \mathrm{~mm}$ diameter with a

148 point angle of $118^{\circ}$ and an helix angle of $30^{\circ}$ was used. The drilling depth was $4 \mathrm{~mm}$.

149 Drill is made of stainless steel with a diamond-like carbon (DLC) coating. The whole

150 machine, sample, and tool are considered perfectly rigid so that the cutting vibrations

151 are negligible.

152 Samples were clamped and mounted on a Kistler $9256 \mathrm{C} 2$ piezoelectric dynamometer

153 to measure the feed force $F$ and the cutting torque $M$, see Fig. 2(b). The signal was

154 amplified by a Kistler 5017B charge amplifier and recorded by a National Instrument

155 data acquisition system coupled with a Labview software. Signals obtained were

156 filtered by a Butterworth type filter, traditionally used during the machining data

157 processing.

158 Temperature data were recorded by two K-type thermocouples OMEGA KMTSS-

159 IM025U-150 with a wire diameter of $0.25 \mathrm{~mm}$ implanted in pre-drilled holes. The 160 measuring junctions were positioned precisely at $1.5 \mathrm{~mm}$ depth and 0.5 and $0.9 \mathrm{~mm}$

161 from the wall, see Fig. 2(c). A conductor paste was injected through the holes to

162 optimise the conduction from bone to thermocouples.

163 After drilling tests, microtomography of drilled bone was performed with the parameters

164 presented previously. The reconstruction process of bone sample volume was

165 conducted via RX Solutions X-ACT software. The resetting of drilled and undrilled bone 
166 tomograms was done on Avizo 9 software. By subtracting tomographic images before and after drilling tests, the 3D volume of the drilling zones of bone was obtained to represent our volume of interest. To facilitate the post-treatment, these zones were approximated by the addition of a cylinder and a cone, Fig. 3(a). Bone volume fractions (BV/TV) were then determined within these zones. BV/TV is extracted from each slice through drilling direction of the whole stack of the volume of interest.

172

174

$$
\Delta T(x, y, z, t)=\frac{q d s}{8 \rho c(\pi a)^{3 / 2}} \int_{0}^{t}\left(t-t^{\prime}\right)^{-3 / 2} e^{-\left\{\left(x-V t^{\prime}\right)^{2}+\left(y-y^{\prime}\right)^{2}+\left(z-z^{\prime}\right)^{2}\right\} / 4 a\left(t-t^{\prime}\right)} d t^{\prime}
$$

\subsection{Analytical modelling of bone temperature rise}

The temperature distribution in the bone can be obtained from the approach developed by Carslaw and Jaeger [23]. A fixed coordinate system $(x, y, z)$ is associated to the bone sample where the beginning of the drilled hole is located at position $x=0$ , Fig. 4. Heat is liberated at time $t^{\prime}$, from 0 to $t\left(0 \leq t^{\prime} \leq t\right)$, at point $Q\left(x^{\prime}, y^{\prime}, z^{\prime}\right)$ where the heat rate $q_{c} d s\left(q_{c}\left(w / m^{2}\right)\right)$ is associated to an elementary surface $d s$ and due to the cutting process. During drilling, the heat source moves with a constant speed $V$ in $x$ direction so that $x^{\prime}=V t^{\prime}$ as shown in Fig. 4. When the bone sample is assimilated to an infinite medium (i.e. the bone is supposed to occupy all the space $-\infty<x, y$ and $z<+\infty)$ and according to Carslaw and Jaeger [23], the temperature elevation $\Delta T$ at a point $M(x, y, z)$ for time $t$ is given by:

where $\rho, k, c$, and $a$ represent respectively the density, the thermal conductivity,

187 the specific heat and the thermal diffusivity. Note that in (1), $q_{c}$ is time independent.

188 According to Tu et al. [24], the thermal properties and the density of pig bone are given by: $k=0.45 \mathrm{~W} / \mathrm{Km}, c=1640 \mathrm{~J} / \mathrm{kg} K$ and $\rho=1640 \mathrm{~kg} / \mathrm{m}^{3}$. 
190 A similar approach has been proposed by Feldmann et al. for the tool tip contribution

$191[19,22]$. However, there is a difference between the two models. Indeed, during the

192 heat source displacement for $0 \leq t^{\prime} \leq t$, its vertical position $x^{\prime}$ was supposed to be equal

193 to $V t$ in Feldmann et al. work $[19,22]$. This is equivalent to consider that the distance

194 between the heat source $Q\left(x^{\prime}, y^{\prime}, z^{\prime}\right)$ and the measurement position $M(x, y, z)$ is

195 constant for $0 \leq t^{\prime} \leq t$.

196 Eq. (1) means that elevation temperature distribution at time $t$ is obtained by adding 197 the rise temperature caused by the amount of heat liberated during the source

198 displacement namely when it was at the positions $\left(x^{\prime}=V t^{\prime}, y^{\prime}, z^{\prime}\right)$ with $0 \leq t^{\prime} \leq t$.

199 By introducing the variable $\tau=t-t^{\prime},(1)$ is reduced to

$$
\Delta T(x, y, z, t)=\frac{q_{c} d s}{8 \rho c(\pi a)^{3 / 2}} e^{-V(x-V t) / 2 a} \int_{0}^{t} \tau^{-3 / 2} e^{-\left(\frac{d^{2}}{4 a \tau}+\frac{V^{2} \tau}{4 a}\right)} d \tau
$$

201 where $d=\sqrt{(x-V t)^{2}+\left(y-y^{\prime}\right)^{2}+\left(z-z^{\prime}\right)^{2}}$ is the distance from the heat source as reported 202 in Fig. 4.

203

204 From the close form of the time integral term, we get:

$$
\Delta T(x, y, z, t)=\frac{q_{c} d s}{8 \rho c \pi a} e^{-V(x-V t) / 2 a} f(d, t)
$$

206 with

$$
f(d, t)=\frac{1}{d}\left\{e^{V d / 2 a} \operatorname{erfc}(d / 2 \sqrt{a t}+V \sqrt{a t} / 2 a)+e^{-V d / 2 a} \operatorname{erfc}(d / 2 \sqrt{a t}-V \sqrt{a t} / 2 a)\right\}
$$

208 The temperature distribution, due to the drill-bit tip contribution, is obtained by adding 209 the temperature rise caused by each elementary heat source $q_{c} d s\left(q\left[w / \mathrm{m}^{2}\right]\right)$ over the 210 disk surface, see Fig. 4(a): 


$$
T_{t i p}(x, y, z, t)=\frac{1}{8 \rho c \pi a} e^{-V(x-V t) / 2 a} \int_{0}^{R} \int_{0}^{2 \pi} q_{c} f(d, t) d \theta r d r
$$

212 where $R$ is the drill bit radius and $d$ is expressed in polar coordinates

$213 d=\sqrt{(x-V t)^{2}+(y-r \cos \theta)^{2}+(z-r \sin \theta)^{2}} . \ln (5)$, the bone is considered as an infinite 214 medium.

215 For drilling operation, the bone can be assimilated to a half space (i.e. the bone sample 216 occupies the half space $x \geq 0$ and its top surface is located at position $x=0$ ). To obtain 217 the temperature distribution in a semi-infinite medium with adiabatic surface, the image 218 sources $q_{i m} d s$ (with $q_{i m}=q_{c}$ ) are introduced as in Komanduri and Hou [25-27]. These 219 fictive sources move with a constant speed $-V$ in $x$-direction such as $q_{i m} d s$ is located 220 at $Q_{i m}\left(x^{\prime}=-V t^{\prime}, y^{\prime}, z^{\prime}\right)$ where heat is liberated at time $t^{\prime}$, from 0 to $t\left(0 \leq t^{\prime} \leq t\right)$, see Fig. $2214(\mathrm{a})$. The temperature distribution due to $Q_{i m}$ is deduced from (5) by replacing $d$ with 222 $d_{i m}=\sqrt{(x+V t)^{2}+(y-r \cos \theta)^{2}+(z-r \sin \theta)^{2}}$ and $V$ with $-V$. Finally, for the bone 223 assimilated to a half space, we obtain:

$$
T_{t i p}(x, y, z, t)=\frac{1}{8 \rho c \pi a}\left\{e^{-V(x-V t) / 2 a} \int_{0}^{R} \int_{0}^{2 \pi} q f(d, t) d \theta r d r+e^{V(x+V t) / 2 a} \int_{0}^{R} \int_{0}^{2 \pi} q f\left(d_{i m}, t\right) d \theta r d r\right\}
$$

The integral terms in (6) are calculated numerically by using Gauss quadrature method.

To account for the friction between the drill-bit side and the drilled hole, the depth $\mathrm{H}$ of the hole is divided into $N$ small differential elements having ring shape. The height of each element $j(1 \leq j \leq N)$ is given by $\Delta h=H / N$, Fig. $4(\mathrm{~b})$.

229 When the drill bit penetrates deeper into the bone, frictional heat is liberated at time $t^{\prime}$ 230 , from $t_{0 j}$ to $t\left(t_{0 j} \leq t^{\prime} \leq t\right)$, at point $Q_{f}\left(x^{\prime}, y^{\prime}, z^{\prime}\right)$ where the heat rate $q_{f} d s\left(q_{f}\left(w / m^{2}\right)\right)$ is 231 associated to an elementary surface $d s=R d \theta \Delta h$, see Fig. 4(b). The heat source moves 232 with a constant speed $V$ in $x$-direction so that $x^{\prime}=V t^{\prime}$ as shown in Fig. 4(b). Following 
233 the drill bit penetration, each source at $Q_{f}$ is activated at $t^{\prime}=t_{0 j}$ with $234 t_{0 j}=j \Delta h / V+R / V \tan (\psi / 2)$. In this equation, the term $R / \tan (\psi / 2)$ represents the height 235 of the drill-bit tip and $\psi$ is the point angle, see Fig. 4(a). Thus, at time $t$, the number of 236 differential elements (elementary rings of Fig. 4(b)) forming the total friction zone is 237 given by the integer part of the ratio $V t / \Delta h$.

238 Applying the foregoing approach, the temperature distribution in the bone (modeled as a half space), due to the side drill bit contribution (frictional heat), is as follows:

$$
T_{\text {side }}(x, y, z, t)=\frac{R \Delta h}{8 \rho c \pi a} \sum_{j}^{N}\left\{e^{-V\left(x+h_{j}-V t\right) / 2 a} \int_{0}^{2 \pi} q_{f} f\left(d, t-t_{o j}\right) d \theta+e^{V\left(x-h_{j}+V t\right) / 2 a} \int_{0}^{2 \pi} q_{f} f\left(d_{i m}, t-t_{o j}\right) d \theta\right\}
$$

242 with

$$
\left\{\begin{array}{l}
h_{j}=(j-1 / 2) \Delta h \\
d=\sqrt{\left(x+h_{j}-V t\right)^{2}+(y-R \cos \theta)^{2}+(z-R \sin \theta)^{2}} \\
d_{i m}=\sqrt{\left(x-h_{j}+V t\right)^{2}+(y-R \cos \theta)^{2}+(z-R \sin \theta)^{2}}
\end{array}\right.
$$

244 The length $h_{j}$ is defined in Fig. 4(b) and the integral terms in (7) are calculated

245 numerically by using Gauss quadrature method. Note that in (7), $q_{f}$ is time 246 independent.

247 The temperature distribution in the bone, resulting from the two contributions, is given 248 by:

$$
T(x, y, z, t)=T_{0}+T_{\text {tip }}(x, y, z, t)+T_{\text {side }}(x, y, z, t)
$$

250 where $T_{0}$ is the initial bone temperature.

251 During cutting operation, the tool-bone interaction changes due to the variation of the 252 bone behavior from the cortical part to the cancellous bone. Consequently to estimate 253 the heat source, we propose to use the average of the maximum and minimum values 
254 of feed force $F$ and cutting torque $M$ denoted by $\bar{F}$ and $\bar{M}$ respectively, see the 255 Results section. Following this approach, the heat source relative to the tool tip is 256 assumed to be constant during drilling, and equal to a fraction $0<\eta<1$ of the mean 257 value of the mechanical energy :

$$
q_{c}=\eta(\bar{F} V+\bar{M} \omega) /\left(\pi R^{2}\right)
$$

259 where $V$ and $\omega$ represent respectively the feed rate and the rotational speed ( $\mathrm{rad} / \mathrm{s})$.

260 Eq. (10) corresponds to a heat source which is time independent as in the present 261 thermal model.

262 The frictional heat induced by the contact between the drill-bit side and the drilled hole 263 is given by:

$$
q_{f}=\mu_{f} p V_{c}
$$

265 with $\mu_{f}$ the friction coefficient, $p$ the contact pressure and $V_{c}$ the cutting velocity $266 \quad\left(V_{c}=\omega R\right)$.

267 It should be noted that before applying the analytical model to analyse the thermal 268 problem in the bone, we have to calibrate the parameters $\eta$ and $p$. The calibration 269 procedure will be presented and discussed in the Discussion section.

\section{RESULTS}

272 Fig. 3(a) shows an example of obtained 3D volume rendering. Each curve reported in

273 Fig. 3(b) joins bone volume fractions determined on each slice of the drilled zones in 274 the drilling direction. The typical evolution of bone volume fractions confirms the drilling 275 of two different types of bone. The relatively flat part of the curve denotes drilling 276 through cortical bone whose bone volume fraction is relatively stable (greater than 0.9).

277 The highly sloped part of bone volume fraction curve represents the entrance into 
278 spongy bone where BV/TV can drop to 0.4 at the end of the drilling process. It is also

279 visible that the bone volume fraction highly varies from a drilled site to another, even 280 inside the cortical part of the bone.

281 The variation of the feed force and cutting torque during drilling is observed in parallel. 282 The curves obtained are reported in Figs. 5(a) and 5(b). The force rapidly increases at 283 the entrance into bone samples to reach a maximum value. Then, the values slightly 284 decrease until the end of the drilling process where the values drop to zero. This 285 diminution trend is similar to the one observed on the BV/TV curves (Fig. 3b). 286 Therefore, it can be deduced that a relationship exists between the evolution of feed 287 force and bone density. Concerning the torques, their evolution with the drilling depth 288 depends on the cutting speed considered. No general trend can be observed.

289 In order to evaluate the heat source at the drill tip, given by Eq. (9), the average values 290 of feed force $\bar{F}$ and cutting torque $\bar{M}$ are extracted on the curve profiles from the 291 drilling maximal and minimum values, see Figs. 5(a) to 5(d). These figures reveal that $292 \bar{F}$ varies only slightly with cutting speed $V_{c}$ while $\bar{M}$ is larger for $V_{c} \geq 10 \mathrm{~m} / \mathrm{min}$ than 293 for $V_{c} \leq 5 \mathrm{~m} / \mathrm{min}$. The obtained values $\bar{F}=18 \mathrm{~N}$ and $\bar{M}=0.08 \mathrm{~N} . \mathrm{m}$ (mean value for $V_{c}$ 294 from 2 to $20 \mathrm{~m} / \mathrm{min}$ ) have been used to estimate the heat source (9).

296 After model calibration (described and discussed in the Discussion section), the 297 predicted temperature of the model and measurement are compared. Fig. 6 shows the comparison for both positions of thermocouples and for each cutting velocity. All curves present two phases with a first one where the bone temperature increases because of 300 the drilling process and a second one illustrating the cooling phase. The culmination of 301 the curve coincides with the end of the drilling stage. 
304 The influence of the heat source due to the lateral friction is reported in Fig. 7(a). As expected, the lateral friction induces an increase in the temperature rise. A modification 306 of the position of the maximum temperature is also observed mainly for the small value 307 of cutting velocity $2 \mathrm{~m} / \mathrm{min}$. Thus, the duration of the temperature increase becomes 308 larger. Fig. 7 (b) shows how the predicted temperature changes when the bone is assimilated to an infinite or semi-infinite medium. When a semi-infinite medium with an

310 adiabatic surface is considered (i.e. the bone sample occupies the half space $x \geq 0$ 311 and its top surface is located at position $x=0$ ), the temperature rises obtained are 312 higher but the global profiles of the temperature curves remain unchanged. Concerning 313 the comparison of the maximal temperature rises, the increase with $V_{c}$ from 2 to 10 $314 \mathrm{~m} / \mathrm{min}$ is reproduced by the model, Fig. 7(c). From 10 to $20 \mathrm{~m} / \mathrm{min}$, the predicted values 315 tend to saturate while the temperature measurements show a decrease, Fig. 7(c).

\section{DISCUSSION}

319 Heat generation, due to the conversion of fraction of cutting energy into thermal energy, 320 is a critical parameter for thermal modelling of bone drilling. As discussed before, the 321 application of the metal machining theory assumes that the cutting mechanisms are 322 similar in bone and metal: in particular, the shear angle can be estimated from the 323 Merchant law [16]. For instance, Davidson and James [11], Maani et al. [12], Lee et al. 324 [8] have supposed that a fraction of shearing energy, due to the primary shear zone, 325 goes into the bone. In Maani et al. [12], Lee et al. [8], this fraction was deduced from 
326 the metal machining theory of Oxley [14] and Davidson and James [11] supposed that 327 it is equal to 0.5 .

328 In the present approach, bone temperature rise results from two main sources: the 329 drill-bit tip (cutting process) and the friction between the drill-bit side and the drilled 330 hole. The values of both sources $q_{c}$ and $q_{f}$ are obtained thanks to relationships (10) 331 and (11). Expression (10) requires the determination of average values of feed force $332 \bar{F}$ and cutting torque $\bar{M}$ which are provided in Fig. 5. As shown in Fig. 5(c), the drilling 333 force $\bar{F}$ is almost constant as a function of the cutting speed. Fig. 5(d) shows two 334 distinct levels for the torque $\bar{M}$ : one for the smallest cutting velocities 2 and $5 \mathrm{~m} / \mathrm{min}$ 335 and a higher value for velocities 10 and $20 \mathrm{~m} / \mathrm{min}$. The values used in this work are: $336 \bar{F}=18 N$ and $\bar{M}=0.08 N . m$ (average value of $\bar{M}$ for $V_{c}$ from 2 to $20 \mathrm{~m} / \mathrm{min}$ ). Further 337 investigations with higher values of cutting velocities would have to be conducted in 338 order to complete these first results. Finally, the determination of $q_{c}$ and $q_{f}$ requires 339 the values of parameters $\mu_{f}, \eta$ and $p$. The value of $\mu_{f}$ was fixed to 0.3 according to 340 data from the literature. Indeed, Rancourt et al. report that $\mu_{f}=0.3$ for tibial cancellous 341 bone sliding against stainless steel surface [28]. Davim and Marques [29] analyzed the 342 influence of sliding velocity on $\mu_{f}$ for bovine bone-steel contact pair. They indicate that $343 \mu_{f}=0.25$ for sliding velocities from $0.02 \mathrm{~m} / \mathrm{s}$ to $0.1 \mathrm{~m} / \mathrm{s}$. More recently, Zhu et al. [30] 344 examined the variation of $\mu_{f}$ in function of contact pressure and sliding velocity with 345 water lubrication for bovine femoral cortical bone-316 L stainless steel contact pair. For 346 sliding velocity of $0.44 \mathrm{~m} / \mathrm{s}$ and contact pressure of $0.42 \mathrm{MPa}$, Zhu et al. [30] showed 347 that $\mu_{f}=0.25$. 
348 Concerning parameters $\eta$ (fraction of mechanical energy resulting from the tool tip349 bone interaction converted into heat) and $p$ (contact pressure between the drill-bit side 350 and the drilled hole), a calibration procedure was carried out. From the parametric 351 study reported in Fig. 7(a), it appears that the time $t_{c}$, corresponding to the maximum 352 temperature rise $\Delta T_{\max }$ ( $t_{c}$ is time during which temperature increases), is mainly 353 dependent on the side heat source (11) (via the contact pressure $p$ ) especially for 354 small cutting velocities. Based on this trend, the calibration of $p$ and $\eta$ was performed 355 with respect to the time $t_{c}$ and $\Delta T_{\max }$ respectively. Thus, the calibration procedure is 356 based on the following iterative approach. First, the two parameters were varied within 357 a defined range (for instance $\eta \in[0.1,0.7]$ and $p[\mathrm{MPa}] \in[0,20]$ ). Secondly, for each value 358 of $\eta$, pressure $p$ is selected so that the condition $t_{c}^{\text {model }} \approx t_{c}^{e x p}$ is satisfied. Finally, among 359 all values of $\eta$ and for each calibrated pressure, the chosen value of $\eta$ must satisfy 360 the condition $\Delta T_{\max }^{\text {model }} \approx \Delta T_{\max }^{e x p}\left(t_{c}^{e x p}\right.$ and $\Delta T_{\max }^{e x p}$ represent the experimental measurements). 361 According to this approach, $\eta$ and $p$ were calibrated such as the experimental 362 temperature and the model prediction are as close as possible for the smallest cutting 363 speed $V_{c}=2 \mathrm{~m} / \mathrm{min}$ as reported in Fig. 6(a) (temperature was measured at $0.5 \mathrm{~mm}$ from 364 the drilled hole, see Fig. 2). The values of parameters $\eta$ and $p$ obtained with this 365 calibration are $\eta=0.35$ and $p=1 M P a$. Besides, Fig. 7 (c) indicates that the difference 366 between the temperature elevation when the bone is assimilated to a semi-infinite 367 medium with adiabatic surface or considered as an infinite medium is more significant 368 for $V_{c}=2 \mathrm{~m} / \mathrm{min}$ than for $V_{c}=20 \mathrm{~m} / \mathrm{min}$. This can be explained by the fact that the feed 369 rate $V$ increases with the cutting speed $V_{c}$ and, at low feed rate, conduction 
overcomes the convection of heat (thanks to the heat source displacement) so that

371 heat diffusion will extend deep into the infinite medium.

372 In order to dissociate the effect of the feed rate per tooth $f_{t}$ from the influence of $V_{c}$,

$373 f_{t}$ is kept constant $f_{t}=0.02$ mm/tooth. Fig. 6. shows the effect of cutting speed $V_{c}$ on

374 the bone temperature elevation over time at depth $1.5 \mathrm{~mm}$ and for the two 375 thermocouples placed at 0.5 and $0.9 \mathrm{~mm}$ from the hole (denoted respectively by ' $\mathrm{T}_{\mathrm{h} 1}{ }^{\prime}$ 376 and 'Th2', see Fig. 2). As expected, the model results indicate that the temperature is 377 an increasing function of $V_{c}$ (i.e. the rotational speed $\omega$ ) while the exposure time 378 decreases with $V_{c}$. This temperature growth is more significant for 'Th1' than for 'Th2'.

379 Overall, the model correctly reproduces the evolution of temperature rise with time $t$ 380 from 0 to $t_{c}$ (i.e. $0 \leq \Delta T \leq \Delta T_{\max }$ ). The low heat conductivity and the high heat capacity 381 of the bone result in lower temperatures at the location of the second thermocouple 382 'Th2', Figs. 6(e)-6(h). Because of keeping $f_{t}$ constant $\left(f_{t}=0.02 \mathrm{~mm} /\right.$ tooth. $)$, the feed 383 rate $V$ increases with cutting speed $V_{c}\left(V=f_{t} V_{c} /(\pi R)\right)$. Consequently, the heat 384 sources increase with $V_{c}$ while the duration of heat exchange (exposure time) with the 385 bone is reduced. These two opposite effects result in a slowing down in the increase 386 of the maximum temperature with $V_{c}$ as reported in Fig. 7(c) (the heat sources $q_{c}$ and $387 q_{f}$ are proportional to $V_{c}$, see Eq. (10) and (11), but the evolution of the maximum 388 temperature results from the two opposite effects). The model predictions reveal that 389 the growth of cutting speed $V_{c}$ from 2 to $5 \mathrm{~m} / \mathrm{min}$ induces an increase of temperature 390 of thermocouple 'Th1' of about $16{ }^{\circ} \mathrm{C}\left(8^{\circ} \mathrm{C}\right.$ for ' $\mathrm{Th2}$ ') while it is about $2^{\circ} \mathrm{C}$ for a variation 391 from 10 to $20 \mathrm{~m} / \mathrm{min}\left(2{ }^{\circ} \mathrm{C}\right.$ for ' $\left.\mathrm{Th} 2^{\prime}\right)$. These tendencies are close to the temperature 392 measurements, Fig. 7(c). It can also be observed from Fig. 7 that the analytical model 393 predicts the maximum temperature rise with a maximum error of $18 \%$ for ' $T \mathrm{~h} 1$ ' whereas 
394 we have $28 \%$ for 'Th2', which is logical as the model was calibrated for the closest 395 position of the thermocouple. The difference between model results and 396 measurements can be attributed to several parameters which are not considered in 397 the present model: (i) the evolution of the bone density during cutting, (ii) the 398 relationship between the bone thermal properties and its density, (iii) the influence of 399 the presence of both thermocouples on the bone temperature distribution, and (iv) the 400 effect of cutting conditions on the model parameters : $\eta, p, \bar{M}$ and $\mu_{f}$.

401 During bone drilling, the temperature rise depends on the bone density (through $402 \mathrm{BV} / \mathrm{TV}$ ). Fig. 3(b) shows that the ratio BV/TV is much higher for the cutting tests with $403 V_{c}=2$ and $5 \mathrm{~m} / \mathrm{min}$ than for $V_{c}=10$ and $20 \mathrm{~m} / \mathrm{min}$. Note also that the thermal conductivity 404 depends on the bone density, see Feldmann et al. [31]. These variations are not taken 405 into account in the present approach where the heat sources are supposed to be 406 constant. To improve the model predictions, the effect of BV/TV on the heat generation 407 and on the thermal conductivity have to be considered. Additionally, the model does 408 not reproduce correctly the cooling stage. Indeed to reduce the computational time, 409 the cooling process was estimated from the moving heat source solution by keeping it 410 to zero during the cooling step (i.e. when $t_{c} \leq t$ ). To reproduce the bone cooling 411 process when $t_{c}<t$, we have to consider the numerical solution of the heat problem 412 where the initial temperature distribution corresponds to the solution given by the 413 present model at $t=t_{c}$.

414 The thermal damage of the bone is mainly dependent on two factors: exposure time 415 and temperature magnitude. Several parameters affect these two critical factors: 416 drilling conditions, drilling strategies, cooling and thermomechanical bone properties. 417 From the foregoing comparison between the model predictions and the experimental 
results, it appears that despite the model simplicity, it can be helpful to examine the bone necrosis in terms of drilling conditions for $0 \leq t \leq t_{c}$ (i.e. $0 \leq \Delta T \leq \Delta T_{\max }$ ).

\section{CONCLUSION}

422 In the present study, a simplified analytical model based on the moving heat source approach is developed. Besides, to be closer to the boundary conditions of the thermal

424 problem, the method of image sources has been applied. Because of the complexity of the chip formation mechanisms in bone cutting process, the heat source due to the drill-bit tip has been supposed to be proportional to the cutting energy. The friction between the drill-bit side and the drilled hole was also taken into account. Drilling experiments were conducted on fresh femur pig bone. The distribution of bone volume fraction through the drilled hole was determined by microtomography for the bone 430 samples. The present model requires the calibration of two parameters. The 431 experimental validation has shown the model reproduces satisfactorily the increase in temperature up to the maximum value while it overestimates the temperature during

433 the cooling stage. A parametric study, concerning the thermal boundaries conditions 434 (infinite or finite medium) and the lateral friction, was also performed. The results show 435 that the difference of temperature rise between semi-infinite or infinite medium is more 436 pronounced for small cutting velocities. The lateral friction has a direct influence on the duration of the temperature increase, especially for small cutting velocities. Finally, the 438 present simplified approach can be improved by considering the effects of the bone 439 volume fraction which can present a significant variation in the bone sample. 
444 ETHICAL APPROVAL: Not required

\section{FUNDING}

447 This work was supported by the French Ministry of Higher Education and Research.

\section{ACKNOWLEDGMENTS}

450 The authors would like to thank Alexis Jacquillard for its contribution to the 451 experimental work.

\section{REFERENCES}

454 1. Pandey RK, Panda SS. Drilling of bone: a comprehensive review. Journal of $455 \quad$ Clinical Orthopaedics and Trauma 2013;4:15-30.

2. Bachus KN, Rondina MT, Hutchinson DT. The effects of drilling force on cortical temperatures and their duration: an in vitro study. Medical Engineering and Physics 2000;22:685-691.

3. Eriksson AR, Albrektsson M. Temperature threshold levels for heat-induced bone tissue injury: a vital-microscopic study in the rabbit. Journal of Prosthetic Dentistry 1983;50:101-107.

4. Lee J, Ozdoganlar OB, Rabin Y. An experimental investigation on thermal exposure during bone drilling. Medical Engineering and Physics 2012;34:15101520.

5. Hillery MT, Shuaib I. Temperature effects on the drilling of human and bovine one. Journal of Materials Processing Technology 1999;92-93:302-308. 
6. Bertollo N, Walsh WR. Drilling of Bone: practicality, limitations and complications associated with surgical drill-bits. In: Biomechanics in Applications 2011;53-83.

7. Augustin G, Davila S, Mihoci K, Udiljak T, Vedrina DS, Antabak A. Thermal osteonecrosis and bone drilling parameters revisited. Archives of Orthopaedic and Trauma Surgery 2007;128:71-77.

8. Lee J, Rabin Y, Ozdoganlar OB. A new thermal model for bone drilling with applications to orthopaedic surgery. Medical Engineering and Physics. 2011;33:1234-1244.

9. Gupta V, Pandey PM. Experimental investigation and statistical modeling of temperature rise in rotary ultrasonic bone drilling. Medical Engineering and Physics 2016;38:1330-1338.

10. Matthews LS, Hirsch C. Temperatures Measured in Human Cortical Bone when Drilling. Journal of Bone and Joint Surgery 1972;54:297 -308.

11. Davidson SRH, James DF. Drilling in Bone: Modeling Heat Generation and Temperature Distribution. Journal of Biomechanical Engineering 2003;125:305314.

12. Maani N, Farhang K, Hodaei M. A Model for the Prediction of Thermal Response of Bone in Surgical Drilling. Journal of Thermal science and Engineering Applications 2014;6: 041005-1-041005-16.

13. Kalidindi V. Optimization of drill design and coolant systems during dental implant surgery, Master's Thesis, University of Kentucky, 2004.

14. Oxley PLB. The Mechanics of Machining: an Analytical Approach to Assessing Machinability. Chichester, E. Horwood ; New York : Halsted Press, 1989.

15. Jacobs $\mathrm{CH}$, Pope MH, Berry JT, Hoaglund F. A study of the bone machining process - Orthogonal cutting. Journal of Biomechanics 1974;7:131-136. 
16. Merchant ME. Mechanics of the metal cutting process. II. Plasticity conditions in orthogonal cutting. Journal of Applied Physics 1945;16:318-324.

17. Wiggins KL, Malkin S. Orthogonal Machining of Bone. Journal of Biomechanical Engineering 1978;100: 122-130.

18. Liao Z, Axinte DA. On chip formation mechanism in orthogonal cutting of bone. International Journal of Machine Tools and Manufacture 2016;102: 41-55.

19. Feldmann A, Ganser P, Nolte L, Zysset P. Orthogonal cutting of cortical bone: Temperature elevation and fracture toughness. International Journal of Machine Tools and Manufacture 2017;1:1-11.

20. Sugita N, Mitsuishi M. Specifications for machining the bovine cortical bone in relations to its microstructure. Journal of Biomechanics 2009;42:2826-2829.

21. Jaeger JC. Moving source of heat and the temperature at sliding contacts. Journal and Proceedings of the Royal Society of New South Wales 1942;76:203-224.

22. Feldmann A, Anso J, Bell B, Williamson T, Gavaghan K, Gerber N, Rohrbach H, Weber S, Zysset P. Temperature prediction model for bone drilling based on density distribution and in vivo experiments for minimally invasive robotic cochlear implantation. Annals of Biomedical Engineering 2016;44:1576-1586.

23. Carslaw HS, Jaeger JC. Conduction of heat in solids. Oxford University Press. 1959.

24. Tu Y, Chen L, Ciou J, Hsiao C. Finite element simulation of bone temperature rise during bone drilling based on a bone analog. Journal of Medical and Biological Engineering 2012;33:269-274.

25. Komanduri R, Hou ZB. Thermal modeling of the metal cutting process. Part-III-temperature-rise-distribution-due-to-the-combined-effects-of-shear-plane-heat- 
source-and-the-tool-chip-interface-frictional-heat-source. International Journal of Mechanical Sciences 2001;43:89-107.

518 26. Komanduri R, Hou ZB. Thermal modeling of the metal cutting process: part I519 temperature rise distribution due to shear plane heat source. International Journal of Mechanical Sciences 2000;42:1715-1752.

27. Komanduri R, Hou ZB. Thermal modeling of the metal cutting process-Part II: temperature rise distribution due to frictional heat source at the tool-chip interface. International Journal of Mechanical Sciences 2001;43:57-88.

28. Rancourt $D$, Shirazi-Adl $A$ A, Drouin G, Paiement G. Friction properties of the interface between porous-surfaced metals and tibial cancellous bone. Journal of Biomedical Materials Research 1990;24:1503-1519.

29. Davim JP, Marques N. Dynamical experimental study of friction and wear behavior of bovine cancellous bone sliding against a metallic counterface in a water lubrificated environment. Journal of Materials Processing Technology 2002;152: 389-394.

30. Zhu B, Xiaofeng $\mathrm{H}$, Zhao T. Friction and wear characteristics of natural bovine bone lubrificated with water. Wear 2015;322:91-100.

31. Feldmann A, Wili P, Maquer G, Zysset P. The thermal conductivity of cortical and cancellous bone. European Cells \& Materials 2018;35:25-33. 